\title{
Are tumor-associated micro-angiogenesis and lymphangiogenesis considered as the novel prognostic factors for patients with Xp11.2 translocation renal cell carcinoma?
}

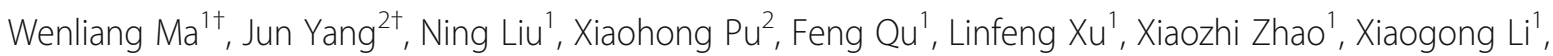
Gutian Zhang ${ }^{1}$, Hongqian Guo', Dongmei Li, ${ }^{3,4}$ and Weidong Gan ${ }^{1 *}$

\begin{abstract}
Background: Tumor micro-angiogenesis and lymphangiogenesis are effective prognostic predictors in many solid malignancies. However, its role on Xp11.2 translocation RCC has not been fully elucidated. Herein, we purposed to explore the correlation between quantitative parameters of tumor-related micro-angiogenesis or lymphangiogenesis and the prognosis of Xp11.2 translocation renal cell carcinoma (Xp11.2 translocation RCC).

Methods: Tissue samples were obtained from 34 Xp11.2 translocation RCC and 77 clear cell renal cell carcinoma (ccRCC) between January 2007 and December 2018. Micro-angiogenesis was detected using CD34 antibody and quantified with microvessel density (MVD) and microvessel area (MVA), while the lymphangiogenesis in RCC was immunostained with D2-40 antibody and assessed using lymphatic vessel density (LVD) and lymphatic vessel area (LVA). The Kaplan-Meier method of survival analysis was used to estimate prognosis, and both univariate and multivariate analysis was performing using the Cox proportional hazards.

Results: The MVD and MVA of Xp11.2 translocation RCC in two detected areas (intratumoral and peritumoral area) were not significantly different from that of cCRCC (all $P>0.05$ ). Notably, D2-40-positive lymphatic vessels of Xp11.2 translocation RCC were highly detected in the peritumoral area compared to the intratumoral area. Interestingly, the peritumoral LVD and LVA of Xp1 1.2 translocation RCC were higher than that of cCRCC (all $P<0.05$ ). Furthermore, both intratumoral MVD or MVA and peritumoral LVD or LVA were significantly associated with pT stage, pN stage, CM stage, AJCC stage, and WHO/ISUP grade (all $P<0.05$ ). Univariate analysis of Cancer-specific survival (CSS) revealed that CSS was substantially longer in patients with low intratumoral MVD or MVA than in patients with high intratumoral MVD or MVA ( $P=0.005$ and $P=0.001$, respectively). Lastly, the Cox proportional hazards model in CSS demonstrated that both intratumoral MVD or MVA and peritumoral LVD or LVA were not independent prognostic parameters (all $P>0.05$ ).
\end{abstract}

(Continued on next page)

\footnotetext{
* Correspondence: gwd@nju.edu.cn

'Wenliang Ma and Jun Yang contributed equally to this work.

'Department of Urology, Nanjing Drum Tower Hospital, The Affiliated

Hospital of Nanjing University Medical School, No. 321 Zhongshan Road,

Nanjing, Jiangsu Province, People's Republic of China 210008

Full list of author information is available at the end of the article
}

(c) The Author(s). 2020 Open Access This article is licensed under a Creative Commons Attribution 4.0 International License, which permits use, sharing, adaptation, distribution and reproduction in any medium or format, as long as you give appropriate credit to the original author(s) and the source, provide a link to the Creative Commons licence, and indicate if changes were made. The images or other third party material in this article are included in the article's Creative Commons licence, unless indicated otherwise in a credit line to the material. If material is not included in the article's Creative Commons licence and your intended use is not permitted by statutory regulation or exceeds the permitted use, you will need to obtain permission directly from the copyright holder. To view a copy of this licence, visit http://creativecommons.org/licenses/by/4.0/ The Creative Commons Public Domain Dedication waiver (http://creativecommons.org/publicdomain/zero/1.0/) applies to the data made available in this article, unless otherwise stated in a credit line to the data. 
(Continued from previous page)

Conclusions: This study outlines that Xp11.2 translocation RCC is a highly vascularized solid RCC, characterized by rich lymph vessels in the peritumoral area. Quantitative parameters of micro-angiogenesis and lymphangiogenesis could not be considered as novel prognostic factors for patients with xp11.2 translocation RCC.

Keywords: Micro-angiogenesis, Lymphangiogenesis, Renal cell carcinoma, Xp11.2 translocation, TFE3, Prognosis

\section{Background}

In primary tumors, distant metastasis is the most important predictive factor of prognosis in many malignant tumors. The common route of tumor metastasis involves tumor cell invasion and penetration lymphatics and/or blood vessels, subsequently disseminating to distant organs. Furthermore, angiogenesis within the tumor is crucial for tumor growth and metastasis, whereas lymphangiogenesis is a critical factor for tumor progression [1-3]. Growth of the primary tumor mass requires angiogenesis, and new microvessels within tumors are predominant sites for tumor cell entry into circulation due to lack of intact basement membrane and a tight junction between endothelial cells [4]. Vascular endothelial growth factors family members stimulate lymphangiogenesis in tumors thereby enhancing metastatic processes $[2,5]$.

Xp11.2 translocation renal cell carcinoma (Xp11.2 translocation $\mathrm{RCC}$ ) is a rare subtype of renal cell carcinoma (RCC), characterized by several different chromosomal translocations involving Xp11.2 and the formation of TFE3 fusion genes, followed by overexpression of TFE3 protein [6]. Patients with Xp11.2 translocation RCC have often presented with advanced stages and demonstrated an invasive clinical course and poor prognosis [7]. In addition, Xp11.2 translocation RCC patients exhibited moderate prolonged enhancement under dynamic contrast-enhanced computed tomography and were more susceptible to lymph node metastasis than those with common subtypes of RCC $[8,9]$. Recent studies based on microvessel density (MVD), microvessel area (MVA), lymph vessel density (LVD), and lymph vessel density (LVA) have revealed that micro-angiogenesis and lymphangiogenesis are significant prognostic predictors of RCC or clear cell renal cell carcinoma (ccRCC) [10-13]. However, the role of micro-angiogenesis and lymphangiogenesis within Xp11.2 translocation RCC has not been fully elucidated. Microvessel density (MVD) or LVD, parameter designed to quantify the extent of tumor vascularization or lymphatics, refers to the number of small blood or lymph vessels per tumor area, while MVA or LVA is described as the total lumen area of small or lymph vessels. Nevertheless, due to limited direct studies on Xp11.2 translocation RCC, we quantified micro-angiogenesis and lymphangiogenesis within Xp11.2 translocation RCC using the parameters including MVD, LVD, MVA, and LVA.
Herein, we purposed to explore the correlation between tumor-related microvascular or lymphatic quantitative parameters and the prognosis of Xp11.2 translocation RCC. Based on quantitatively analyzing the distribution of microvessels and lymphatics of Xp11.2 translocation RCC histologically, and comparing it with ccRCC.

\section{Methods \\ Patients}

This study was reviewed and approved by the institutional ethics committees of Nanjing Drum Tower Hospital. We reviewed the data of 34 patients diagnosed with Xp11.2 translocation RCC from January 2007 to December 2018, while 77 patients with ccRCC were grouped as the control using the statistic method of propensity score matching (PSM) to keep the homogeneity of the clinicopathological data. The Xp11.2 translocation RCC was diagnosed using both TFE3 immunohistochemistry staining and self-designed break-apart TFE3 fluorescence in situ hybridization probes [14]. All enrolled patients were subjected to radical nephrectomy and did not receive neoadjuvant therapy or adjuvant chemotherapy or radiotherapy. Then, the postoperative pathological staging was assessed using the eighth edition TNM classification issued by the AJCC Staging in 2017. The nuclear grade was determined using the 2016 WHO/ISUP pathological nuclear grading system, whereas clinical staging was based on the findings of preoperative ultrasonography, nuclear medicine bone scanning, computed tomography of the abdomen, and lung radiography. Finally, computed tomography of the lung and brain in conjunction with magnetic resonance imaging of the bone and abdomen were performed as necessary [13]. Integrated survival data and tissue samples were available for all 111 patients enrolled in this study.

\section{Immunohistochemistry}

Formalin-fixed paraffin-embedded tissue sections (thickness $4 \mu \mathrm{m}$ ) were deparaffinized in xylene and rehydrated in graded ethanol series. Next, the deparaffinized slides were placed in boiling saline sodium citrate to repair antigen for $10 \mathrm{~min}$. Then, the specimens were incubated in $3 \%$ hydrogen peroxide for 10 min to block endogenous peroxidase activity and also washed with phosphate- 
buffered saline (PBS) three times. Afterward, all dried sections were incubated in $10 \%$ bull serum albumin for $30 \mathrm{~min}$, followed by incubation overnight at $4{ }^{\circ} \mathrm{C}$ with primary CD34 monoclonal antibody (Dako) at 1 : 100 dilution or D2-40 monoclonal antibody (Dako) at 1:100 dilution. The biotinylated secondary antibodies were labeled using the streptomycin avidin-peroxidase solution (Dako) at 1:500 dilution. Lastly, they were stained using Simple DAB Stain Kit (Dako) as per the manufacturer's instructions, and the sections were counterstained with hematoxylin. The sections from each specimen (processed with nonimmunized mouse serum instead of the primary antibody) were used as negative controls.

\section{Quantification of D2-40 positive lymph vessels and CD34 positive microvessels}

To quantify the MVD, tumor sections stained with CD34 antibody were screened to select the maximum staining area (hot spot) by two experienced pathologists based on the criteria [13] at $\times 100$ magnification (Microscope Product ID 7C86158, Model CX23LEDRFS1C, Olympus Corpn., China). Next, the mean value of CD34 positive microvessels within five "hot spots" for each tumor section was computed at $\times 400$ magnification. Microvessel density (MVD) was defined as the number of microvessels within 0.2 square millimeters $\left(\mathrm{mm}^{2}\right)$. Then, 5 consecutive fields within the "hot spots" at $x$ 400 magnification were captured using a digital image capture device (Microscope digital imaging system, Model DM500, Beijing Jiayuan Industrial Technology Co. LTD., China) for the estimation of MVA. The images resolution is 2592 Pixel Per Inch at width and 1944 Pixel Per Inch at height, and it is unchanged during image processing. Additionally, the MVA was calculated using image analysis software (Image-Pro Plus 6.0, Media Cybernetics, USA) and depicted as the mean lumen area of the microvessels within the five $0.20 \mathrm{~mm}^{2}$ fields. The evaluation protocol of LVA and LVD was similar to that of quantifying the microvessels. Finally, intratumoral microvessels and lymphatics were described as those located within the tumor mass, while peritumoral microvessels and lymphatics were outlined as those located within $2 \mathrm{~mm}$ outside of the tumor border.

\section{Statistical analysis}

Quantitative data were expressed as mean \pm standard deviation and compared using Student'st-test, whereas qualitative data were presented as counts and percentages, and assessed using either Chi-Square test or Fisher 's exact test. To control the homogeneity of the clinicopathological data between the two groups, the PSM was applied to balance the clinicopathological covariates including laterality, pathological $\mathrm{T}$ or $\mathrm{N}$ stage, clinical $\mathrm{M}$ stage, AJCC stage, WHO/ISUP grade and tumor size. Moreover, we excluded the age and gender of patients between subgroups when performing PSM since they are different naturally [15]. The Kaplan-Meier method of survival analysis was used to estimate cancer-specific survival (CSS), while statistical comparisons were assessed using the log-rank test. The CSS was described as the time interval between the date of surgery and the date of death or last follow-up. Then, the Cox proportional hazards model was used to evaluate the predictive role of the factors that indicated significance in the longrank test. The significance level was set at $P<0.05$. All statistical analysis was implemented using SPSS software version 23.0 (IBM SPSS Inc., Chicago, IL, USA). The survival curves were drawn using GraphPad Prism software version 7.0.

\section{Results}

\section{Clinicopathological data}

The clinicopathological features of the two groups (Xp11.2 translocation RCC and ccRCC groups) are shown in Table 1 . The mean age was $29.9 \pm 13.5$ years (ranging from 3 to 64 years) in the Xp11.2 translocation RCC group (male to female ratio of 1:1.62), whereas in the ccRCC group (male to female ratio of 1:0.33) it was $57.7 \pm 12.9$ years (ranging from 24 to 82 years). Then, the median follow-up time for the Xp11.2 translocation RCC was 63 months (ranging from 15 to 137 months), while the ccRCC group recorded 53 months (ranging from 8 to 94 months). Notably, there was a statistical difference in the age and gender between subgroups (both $P<0.001$ ). However, after performing PSM there was no significant difference between the two groups in terms of laterality, pathological $\mathrm{T}$ or $\mathrm{N}$ stage, clinical M stage, AJCC stage, WHO/ISUP grade and tumor size (all $P>0.05$ ).

\section{Distribution of microvessels and lymphatic vessels in Xp11.2 translocation RCC}

The CD34-positive microvessels were examined microscopically throughout the intratumoral and peritumoral area of Xp11.2 translocation RCC and ccRCC (Fig. 1a, b and 2a, b). Plenty of CD34-positive microvessels in the intratumoral and peritumoral area were counted (Table 2), but the MVD and MVA of Xp11.2 translocation RCC in the two detected areas (intratumoral and peritumoral area) were not significantly different from those of ccRCC (all $P>0.05$ ). Furthermore, in particular, the MVD and MVA of the intratumoral area in Xp11.2 translocation RCC were significantly different compared to those of the peritumoral area (both $P<0.05$ ). On the other hand, D240-positive lymphatic vessels were assessed 
Table 1 Clinicopathological features of patients with both Xp11.2 translocation RCC and cCRCC

\begin{tabular}{|c|c|c|c|}
\hline \multirow[t]{2}{*}{ Characteristics } & $\begin{array}{l}\text { Xp11.2 translocation } \\
\text { RCC }\end{array}$ & ccRCC & \multirow[t]{2}{*}{$P$-value } \\
\hline & No. (\%) & No. (\%) & \\
\hline Age & & & $<0.001$ \\
\hline$<45$ & $28(82.4)$ & $14(18.2)$ & \\
\hline$\geq 45$ & $6(17.6)$ & $63(81.8)$ & \\
\hline Gender & & & $<0.001$ \\
\hline Male & $13(38.2)$ & $58(75.3)$ & \\
\hline Female & $21(61.8)$ & $19(24.7)$ & \\
\hline Laterality & & & 0.093 \\
\hline Right & $20(58.8)$ & $32(41.6)$ & \\
\hline Left & $14(41.2)$ & $45(58.4)$ & \\
\hline Pathological T stage & & & 0.654 \\
\hline $\mathrm{pT} 1 / \mathrm{pT} 2$ & $29(85.3)$ & $63(81.8)$ & \\
\hline pT3/pT4 & $5(14.7)$ & $14(18.2)$ & \\
\hline Pathological N stage & & & 0.251 \\
\hline $\mathrm{pN}_{0}$ & $27(79.4)$ & 69 (89.6) & \\
\hline $\mathrm{pN}_{1}$ & $7(20.6)$ & $8(10.4)$ & \\
\hline Clinical M stage & & & 0.999 \\
\hline$C M_{0}$ & $32(94.1)$ & $71(92.2)$ & \\
\hline $\mathrm{CM}_{1}$ & $2(5.9)$ & $6(7.8)$ & \\
\hline AJCC stage & & & 0.982 \\
\hline$|/| \mid$ & $27(79.4)$ & $61(79.2)$ & \\
\hline III/IV & $7(20.6)$ & $16(20.8)$ & \\
\hline WHO/ISUP grade & & & 0.401 \\
\hline $1 / 2$ & $24(70.6)$ & $48(62.3)$ & \\
\hline $3 / 4$ & $10(29.4)$ & $29(37.7)$ & \\
\hline Tumor size (cm) & $5.5 \pm 2.7$ & $5.6 \pm 2.3$ & 0.806 \\
\hline
\end{tabular}

Xp11.2 translocation RCC Xp11.2 translocation renal cell carcinoma, ccRCC Clear cell renal cell carcinoma, AJCC American Joint Committee On Cancer, WHO/ ISUP World Health Organization/International Society of Urological Pathology

microscopically throughout the two detected areas of Xp11.2 translocation RCC and ccRCC (Fig. 1c, d and 2c, d). Afterward, D2-40-positive lymphatic vessels in the peritumoral area were detected in all Xp11.2 translocation RCC specimens, while the same vessels in the intratumoral area were recorded in only 9 (26.5\%) Xp11.2 translocation RCC cases. The values presented in Table 2 also demonstrated that the peritumoral LVD and LVA of Xp11.2 translocation RCC were higher than those of ccRCC (all $P<0.05$ ). Nonetheless, the intratumoral LVD and LVA of Xp11.2 translocation RCC were not statistically different compared to that of ccRCC (both $P>0.05$ ). Lastly, the peritumoral LVD and LVA in Xp11.2 translocation RCC were significantly higher than those of the intratumoral area (both $P<0.05$ ) (Table 2).
Association between pathological variables and MVD, MVA, LVD or LVA

As shown in Table 3, the MVD and MVA (but not the LVD or LVA) of the intratumoral area was significantly associated with pT stage $(P=0.023$ and $P<0.001$, respectively), $\mathrm{pN}$ stage (both $P<0.001)$, cM stage $(P=$ 0.017 and $P=0.001$, respectively), AJCC stage (both $P<$ $0.001)$, and $\mathrm{WHO} / \mathrm{ISUP}$ grade $(P=0.008$ and $P=0.002$, respectively). Although we also analyzed the correlation of pathological variables with MVD or MVA in the peritumoral area, no correlation was noted (all $P>0.05$ ) (Table 4). Conversely, the values presented in Table 4 revealed that the peritumoral LVD or LVA was significantly associated with several pathological variables namely pT stage $(P=0.004$ and $P=0.001$, respectively), $\mathrm{pN}$ stage $(P=0.001$ and $P=0.011$, respectively), cM stage $(P=0.009$ and $P=0.002$, respectively), AJCC stage ( $P=0.001$ and $P=0.011$, respectively), and WHO/ISUP grade $(P=0.007$ and $P=0.002$, respectively).

\section{Correlation between clinical prognosis and MVD, MVA, LVD, and LVA}

The threshold level of MVD, MVA, LVD, or LVA was characterized according to the median. The median cutoff values for intratumoral MVD and MVA were 157 and 44,259, while for peritumoral LVD and LVA were 10 and 3326, respectively. High and low group values were defined based on the median cut-off value. Kaplan-Meier analysis of CSS in patients with intratumoral MVD or MVA (Fig. 3a and b) revealed that CSS was significantly longer in patients with low intratumoral MVD or MVA than in patients with high intratumoral MVD or MVA $(P=0.001$ and $P=0.005$, respectively). On the other hand, peritumoral LVD or LVA was not associated with CSS in patients with both low peritumoral LVD or LVA and high peritumoral LVD or LVA in the Fig. $3 c$ and $d(P=0.352$ and $P=0.264$, respectively). Lastly, multivariate analysis using the Cox proportional hazards model for CSS in Table 5 elucidated that both intratumoral MVD or MVA, and peritumoral LVD or LVA were not independent prognostic factors (all $P>0.05$ ).

\section{Discussion}

Angiogenesis and lymphangiogenesis within malignant tumors are crucial for tumor growth and metastasis $[1$, 2]. Numerous studies have confirmed that tumor microangiogenesis and lymphangiogenesis could be effective prognostic predictors in many malignant tumors such as prostatic adenocarcinoma, thyroid cancer, and breast cancer [16-18]. On the contrary, other studies based on meta-analysis showed that tumor micro-angiogenesis and lymphangiogenesis are not useful predictive factors for the prognosis in RCC $[19,20]$. However, Xp11.2 

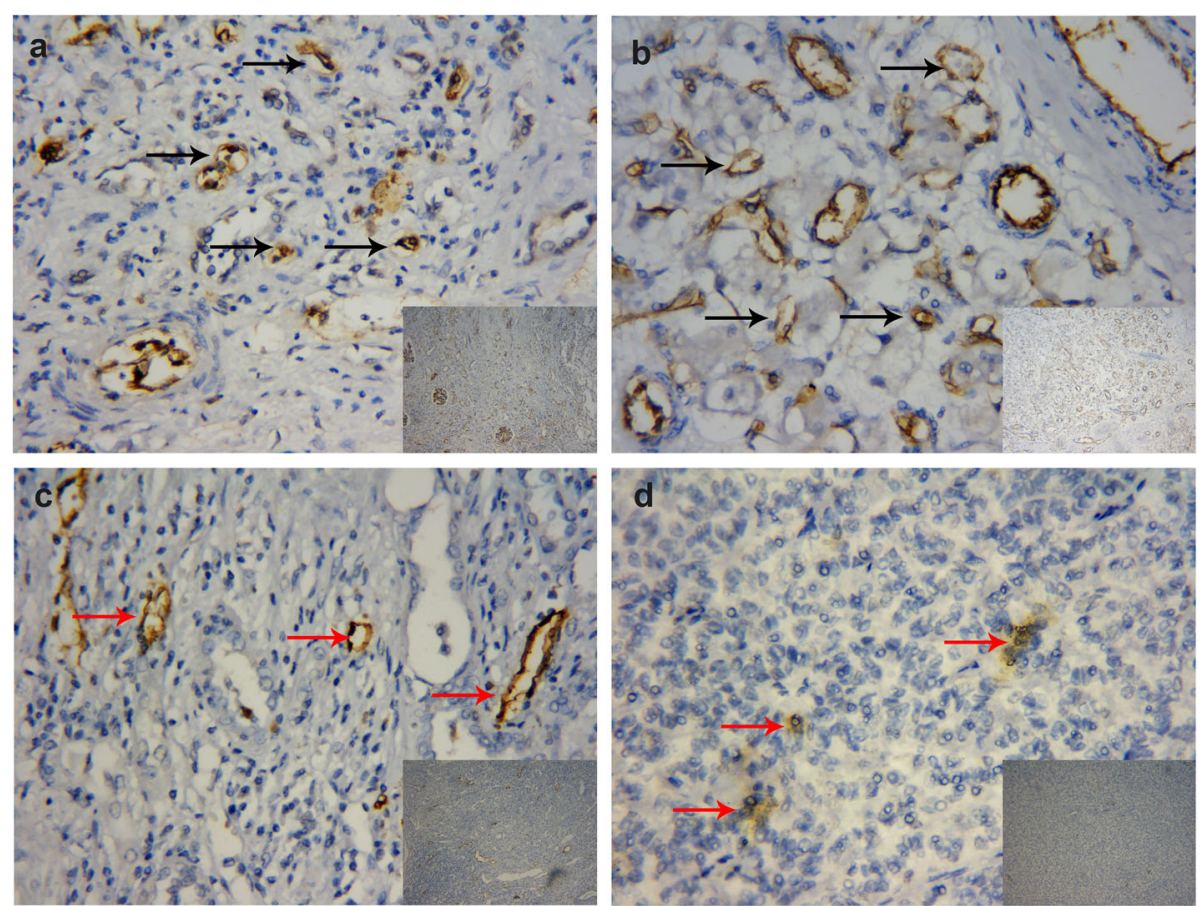

Fig. 1 Representative immunostaining for CD34-positive microvessels (a, b, black arrowheads) and D2-40-positive lymphatic vessels (c, d, red arrowheads) at $\times 100$ and $\times 400$ magnification in Xp11.2 translocation renal cell carcinoma. a Peritumoral microvessels, $\mathbf{b}$ Intratumoral microvessels, c Peritumoral lymphatic vessels, and $\mathbf{d}$ Intratumoral lymphatic vessels
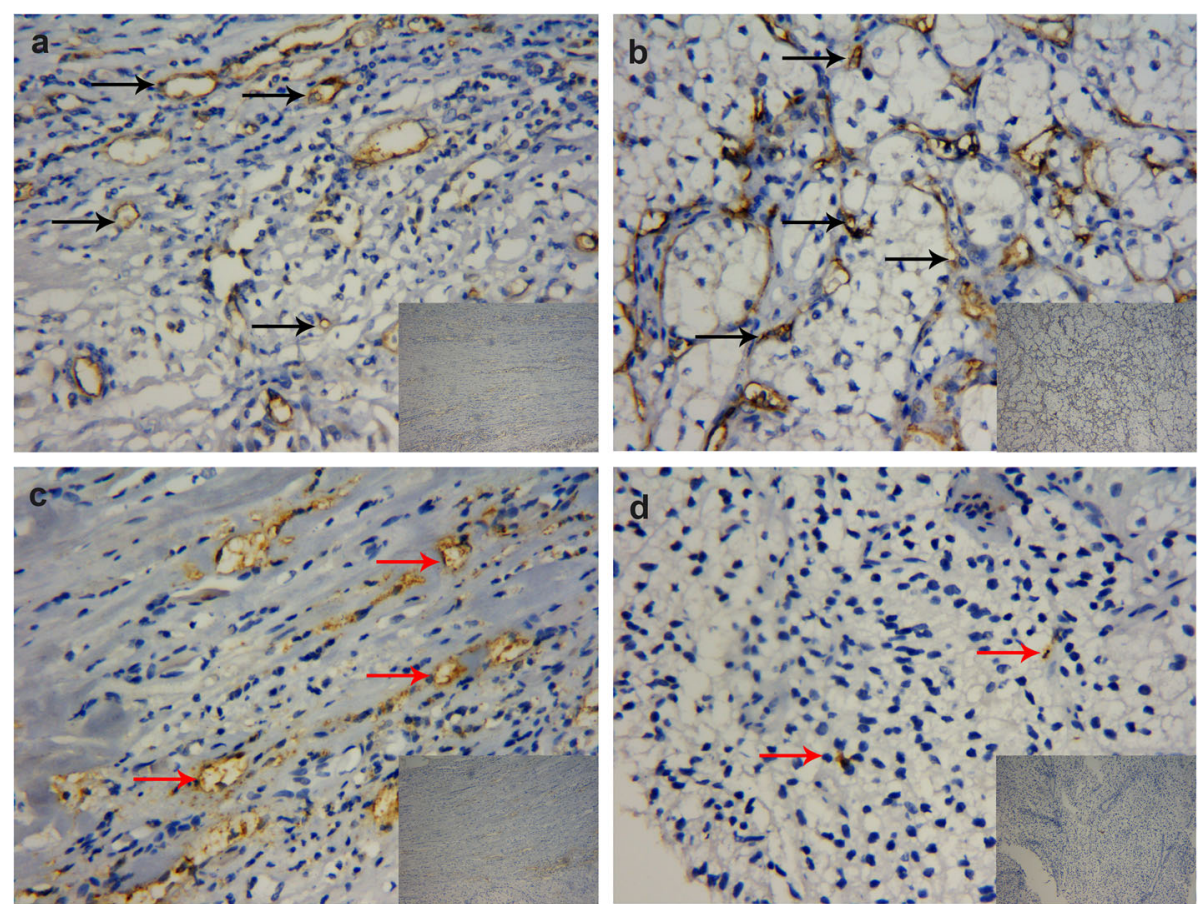

Fig. 2 Representative immunostaining for CD34-positive microvessels ( $\mathbf{a}, \mathbf{b}$, black arrowheads) and D2-40-positive lymphatic vessels (c, $\mathbf{d}$, red arrowheads) at $\times 100$ and $\times 400$ magnification in clear cell renal cell carcinoma. a Peritumoral microvessels, $\mathbf{b}$ Intratumoral microvessels, $\mathbf{c}$ Peritumoral lymphatic vessels, and $\mathbf{d}$ Intratumoral lymphatic vessels 
Table 2 Distribution characteristics of microvessels and lymphatic vessels in both Xp11.2 translocation RCC and cCRCC

\begin{tabular}{|c|c|c|c|c|}
\hline \multirow[t]{2}{*}{$\begin{array}{l}\text { Histological } \\
\text { subtypes }\end{array}$} & & \multirow{2}{*}{$\begin{array}{l}\text { Xp11.2 translocation } \\
\text { RCC } \\
\text { Mean } \pm \text { SD }\end{array}$} & \multirow{2}{*}{$\begin{array}{l}\text { ccRCC } \\
\text { Mean } \pm \text { SD }\end{array}$} & \multirow[t]{2}{*}{$P$-value } \\
\hline & & & & \\
\hline \multirow[t]{4}{*}{ Intra-tumor } & MVD & $157 \pm 10^{*}$ & $153 \pm 12^{*}$ & 0.723 \\
\hline & MVA & $44,259 \pm 8701^{*}$ & $45,665 \pm 10559^{*}$ & 0.649 \\
\hline & LVD & $1 \pm 2^{*}$ & $2 \pm 2^{*}$ & 0.692 \\
\hline & LVA & $535 \pm 563^{*}$ & $481 \pm 465^{*}$ & 0.680 \\
\hline \multirow[t]{4}{*}{ Peri-tumor } & MVD & $108 \pm 10$ & $110 \pm 11$ & 0.247 \\
\hline & MVA & $34,975 \pm 6131$ & $37,009 \pm 6841$ & 0.362 \\
\hline & LVD & $10 \pm 3$ & $8 \pm 3$ & 0.040 \\
\hline & LVA & $3326 \pm 1568$ & $2556 \pm 1120$ & 0.029 \\
\hline
\end{tabular}

Xp11.2 translocation RCC Xp11.2 translocation renal cell carcinoma, ccRCC Clear cell renal cell carcinoma, MVD Microvascular density, MVA Microvascular area, LVA Lymph vessel area, LVD Lymph vessel density, SD Standard deviation; "showed the difference of parameters between intra-tumor and peri-tumor, $P<0.05$

translocation RCC is a rare subtype of RCC, and its clinical outcome is significantly different from the common subtypes of RCC. The Xp11.2 translocation RCC has been noted to depict a wide variation in biological behavior and clinical outcome [7]. Therefore, the role of micro-angiogenesis and lymphangiogenesis within Xp11.2 translocation RCC is imperative, and necessities further studies. Although this study has demonstrated that tumor-related microvascular and lymphatic parameters were not independent prognostic factors for Xp11.2 translocation RCC patients, we revealed that Xp11.2 translocation RCC is a highly vascularized solid RCC, which is characterized by rich lymph vessels in the peritumoral area. This valuable information may provide a histological theoretical basis for tumor metastatic pathways and thereby giving new strategies for treating Xp11.2 translocation RCC based on the tumor antiangiogenic therapy theory.

The tumor micro-angiogenesis has been measured in several ways including MVD, MVA, quantifying tumor angiogenic molecules, microvessel invasion and assessing the presence of tumor-associated angiogenic receptors, while the lymphangiogenesis has been quantified using various methods such as LVD, LVA, lymphatic vessel invasion, and vascular endothelial growth factors family members [10, 12, 21-23]. Currently, the MVD, MVA, LVD, and LVA have widely been used as measurement parameters for assessing micro-angiogenesis and lymphangiogenesis within RCC. In this context, we computed the microvessels and lymphatics within the Xp11.2 translocation RCC using MVD, MVA, LVD, and LVA. Previous studies have opined that there were many vascular markers such as factor VIII, CD31, CD34, and CD105 for marking microvessels endothelium in malignant tumors [12]. Overall, findings from multiple studies have noted that CD34 expression exhibited no variation over time and thus better compared to several other markers for staining microvessels [24, 25]. Herein, CD34 was used as the most suitable vascular markers for counting microvessels in Xp11.2 translocation RCC. Furthermore, other several lymphatic vessel markers namely

Table 3 Relationship between MVD, MVA, LVD or LVA and pathological variables in intratumoral area of Xp11.2 translocation RCC

\begin{tabular}{|c|c|c|c|c|c|c|c|c|c|}
\hline \multirow[t]{2}{*}{ Variables } & \multirow[t]{2}{*}{ No. } & \multicolumn{8}{|c|}{ Intra-tumor (Mean \pm SD) } \\
\hline & & MVD & $P$-value & MVA & $P$-value & LVD & $P$-value & LVA & $P$-value \\
\hline Pathological T stage & & & 0.023 & & $<0.001$ & & 0.718 & & 0.664 \\
\hline $\mathrm{pT} 1 / \mathrm{pT} 2$ & 29 & $156 \pm 10$ & & $41,933 \pm 6967$ & & $1 \pm 2$ & & $553 \pm 602$ & \\
\hline pT3/pT4 & 5 & $166 \pm 4$ & & $57,750 \pm 4244$ & & $1 \pm 1$ & & $431 \pm 260$ & \\
\hline Pathological N stage & & & $<0.001$ & & $<0.001$ & & 0.615 & & 0.911 \\
\hline $\mathrm{pN}_{0}$ & 27 & $154 \pm 9$ & & $41,212 \pm 6658$ & & $1 \pm 2$ & & $529 \pm 604$ & \\
\hline $\mathrm{pN}_{1}$ & 7 & $168 \pm 5$ & & $56,008 \pm 4630$ & & $2 \pm 2$ & & $557 \pm 408$ & \\
\hline Clinical M stage & & & 0.017 & & 0.001 & & 0.690 & & 0.837 \\
\hline $\mathrm{CM}_{0}$ & 32 & $156 \pm 10$ & & $43,329 \pm 8083$ & & $1 \pm 2$ & & $540 \pm 575$ & \\
\hline $\mathrm{CM}_{1}$ & 2 & $168 \pm 1$ & & $59,139 \pm 1477$ & & $1 \pm 1$ & & $454 \pm 445$ & \\
\hline AJCC stage & & & $<0.001$ & & $<0.001$ & & 0.615 & & 0.911 \\
\hline$|/| \mid$ & 27 & $154 \pm 9$ & & $41,212 \pm 6658$ & & $1 \pm 2$ & & $529 \pm 604$ & \\
\hline III/IV & 7 & $168 \pm 5$ & & $56,008 \pm 4630$ & & $2 \pm 2$ & & $557 \pm 408$ & \\
\hline WHO/ISUP grade & & & 0.008 & & 0.002 & & 0.891 & & 0.710 \\
\hline $1 / 2$ & 24 & $154 \pm 9$ & & $41,463 \pm 7031$ & & $1 \pm 2$ & & $559 \pm 635$ & \\
\hline $3 / 4$ & 10 & $164 \pm 9$ & & $50,969 \pm 8965$ & & $2 \pm 1$ & & $478 \pm 358$ & \\
\hline
\end{tabular}

MVD Microvascular density, MVA Microvascular area, LVA Lymph vessel area, LVD Lymph vessel density, AJCC American Joint Committee On Cancer, WHO/ISUP World Health Organization/International Society of Urological Pathology, Xp11.2 translocation RCC Xp11.2 translocation renal cell carcinoma 
Table 4 Relationship between MVD, MVA, LVD or LVA and clinicopathological variables in peritumoral area of Xp11.2 translocation RCC

\begin{tabular}{|c|c|c|c|c|c|c|c|c|c|}
\hline \multirow[t]{2}{*}{ Variable } & \multirow[t]{2}{*}{ No. } & \multicolumn{8}{|c|}{ Peri-tumor (Mean \pm SD) } \\
\hline & & MVD & $P$-value & MVA & $P$-value & LVD & $P$-value & LVA & $P$-value \\
\hline Pathological T stage & & & 0.399 & & 0.563 & & 0.004 & & $<0.001$ \\
\hline $\mathrm{pT} 1 / \mathrm{pT} 2$ & 29 & $107 \pm 10$ & & $34,718 \pm 6183$ & & $9 \pm 3$ & & $2914 \pm 1125$ & \\
\hline pT3/pT4 & 5 & $111 \pm 8$ & & $36,468 \pm 6259$ & & $13 \pm 3$ & & $5719 \pm 1746$ & \\
\hline Pathological N stage & & & 0.397 & & 0.569 & & 0.001 & & 0.011 \\
\hline $\mathrm{pN}_{0}$ & 27 & $106 \pm 10$ & & $34,201 \pm 6083$ & & $9 \pm 3$ & & $2837 \pm 1089$ & \\
\hline $\mathrm{pN}_{1}$ & 7 & $114 \pm 8$ & & $37,960 \pm 5767$ & & $13 \pm 3$ & & $5212 \pm 1778$ & \\
\hline Clinical M stage & & & 0.419 & & 0.175 & & 0.009 & & 0.002 \\
\hline$C M_{0}$ & 32 & $108 \pm 10$ & & $34,713 \pm 6223$ & & $9 \pm 3$ & & $3125 \pm 1381$ & \\
\hline $\mathrm{CM}_{1}$ & 2 & $114 \pm 4$ & & $39,169 \pm 1592$ & & $13 \pm 2$ & & $6539 \pm 356$ & \\
\hline AJCC stage & & & 0.059 & & 0.151 & & 0.001 & & 0.011 \\
\hline$|/| \mid$ & 27 & $106 \pm 10$ & & $34,201 \pm 6083$ & & $9 \pm 3$ & & $2837 \pm 1089$ & \\
\hline III/IV & 7 & $114 \pm 8$ & & $37,960 \pm 5767$ & & $13 \pm 3$ & & $5212 \pm 1778$ & \\
\hline WHO/ISUP grade & & & 0.103 & & 0.181 & & 0.007 & & 0.002 \\
\hline $1 / 2$ & 24 & $106 \pm 10$ & & $34,059 \pm 6357$ & & $9 \pm 3$ & & $2822 \pm 1146$ & \\
\hline $3 / 4$ & 10 & $112 \pm 9$ & & $37,174 \pm 5189$ & & $12 \pm 3$ & & $4536 \pm 1833$ & \\
\hline
\end{tabular}

MVD Microvascular density, MVA Microvascular area, LVA Lymph vessel area, LVD Lymph vessel density, AJCC American Joint Committee On Cancer, WHO/ISUP World Health Organization/International Society of Urological Pathology, Xp11.2 translocation RCC Xp11.2 translocation renal cell carcinoma

D2-40, lymphatic vessel endothelial hyaluronan receptors, and vascular endothelial growth factor receptors are available for detecting lymphatic endothelium [10, 17]. In particular, the D2-40 antibody was used to mark lymphatic vessels in this study due to its specificity for lymphatic vessels [26].

Here, the prognostic value of MVD and MVA indicating the microvessels in RCC remained inconsistent and unclear. In another study by Sharma et al. reported that the MVD within RCC was not significantly correlated with tumor grade and stage, and thereby MVA appeared to be a good prognostic factor for RCC [12]. Elsewhere, multiple studies demonstrated that MVD in RCC exhibited an inverse association with tumor aggressiveness $[24,27]$, whereas other reports revealed no relationship between MVD and survival $[19,20]$. In this work, the intratumoral MVD and MVA were significantly associated with grade and stage of Xp11.2 translocation RCC. However, the intratumoral MVD and MVA were not independent prognostic factors for Xp11.2 translocation RCC. The discrepancy between the results of these studies could be attributed to various factors such as tumor vascular system complexity, pathological subtypes of RCC, sample size, selection of vascular markers, immunohistochemistry staining quality, and methods of microvessel count. Despite different kinds of research focused on the tumor micro-angiogenesis in RCC, the prognostic value of LVD and LVA for assessing the lymphangiogenesis in RCC are rarely studied. Moreover, Iwata et al. reported that both intratumoral and peritumoral LVD were not associated with the pathological features in RCC [10]. On the other hand, a recent study has elucidated that the presence of intratumoral lymphatic vessels exhibited a significant correlation with distant metastasis and lymph node metastasis [28]. In contrast, findings from our study demonstrated that peritumoral LVD or LVA of Xp11.2 translocation RCC was significantly associated with several pathological variables including $\mathrm{pT}$ stage, $\mathrm{pN}$ stage, $\mathrm{cM}$ stage, AJCC stage, and WHO/ISUP grade, while increased peritumoral LVD or LVA indicated a relationship with high pathological stage and increasing nuclear grade. Notably, pathological subtypes of RCC, sample size, immunohistochemistry staining quality, and methods of microvessel count may be major reasons why conclusions from the above studies were very different. Therefore, the role of tumor lymphangiogenesis in the intratumoral and peritumoral area of RCC necessitates further research.

To avoid the selection bias of different areas of the tumor examined under the microscope, we analyzed the intratumoral and peritumoral areas of Xp11.2 translocation RCC. The results revealed that MVD or MVA in the intratumoral area was significantly higher compared to that of the peritumoral area, which was inconsistent with the findings of Cao et al. [29]. In particular, their study implied that the peritumoral area in ccRCC included considerably more microvessels. The inconsistency between these two studies is likely due to pathological subtypes of RCC and microvessel counting methods. Additionally, CSS in Xp11.2 translocation RCC 

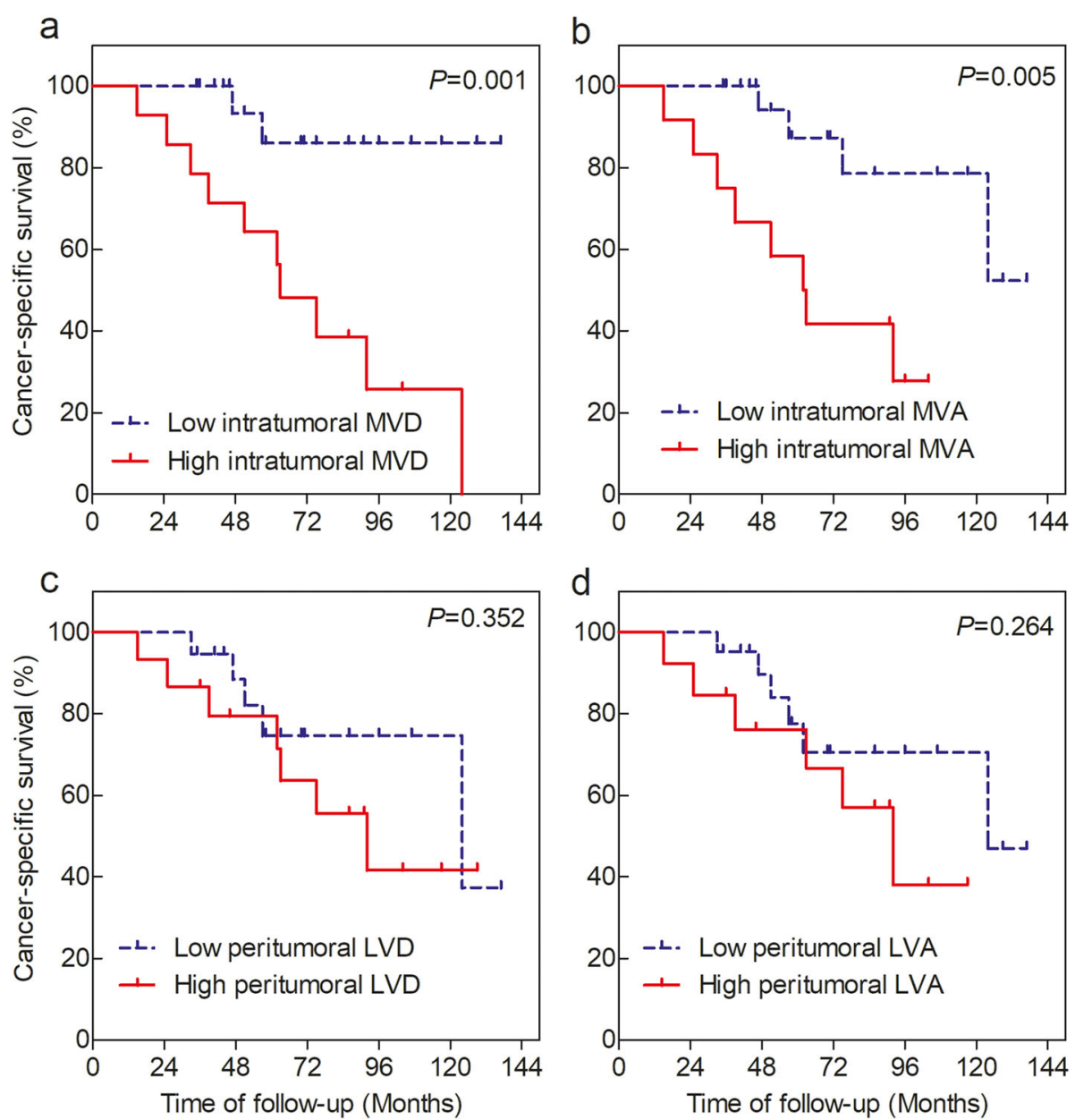

Fig. 3 Kaplan-Meier method plotting cancer-specific survival (CSS) curves in Xp11.2 translocation renal cell carcinoma patients grouped by intratumoral MVD, intratumoral MVA, peritumoral LVD, and peritumoral LVA, stratified according to the median cut-off values. a CSS for patients with low and high intratumoral MVD, $\mathbf{b}$ CSS for patients with low and high intratumoral MVA, $\mathbf{c}$ CSS for patients with low and high peritumoral LVD, and $\mathbf{d}$ CSS for patients with low and high peritumoral LVA

Table 5 Univariate and multivariate analysis of variables predicting CSS

\begin{tabular}{|c|c|c|c|c|c|c|}
\hline \multirow[t]{3}{*}{ Variables } & \multicolumn{6}{|l|}{ CSS } \\
\hline & \multicolumn{3}{|c|}{ Univariate analysis } & \multicolumn{3}{|c|}{ Multivariate analysis } \\
\hline & $\overline{H R}$ & $95 \% \mathrm{Cl}$ & $P$-value & HR & $95 \% \mathrm{Cl}$ & $P$-value \\
\hline AJCC stage (I/II vs III/IV) & 5.595 & $1.697-18.449$ & 0.005 & 5.381 & $1.157-16.858$ & 0.039 \\
\hline WHO/ISUP grade (1/2 vs 3/4) & 3.303 & $1.039-10.505$ & 0.043 & 0.292 & $0.017-5.073$ & 0.398 \\
\hline Intratumoral MVD (< 157 vs $\geq 157$ ) & 7.989 & $1.748-36.502$ & 0.007 & 6.906 & $0.948-50.329$ & 0.057 \\
\hline Intratumoral MVA (<44,259 vs $\geq 44,259)$ & 5.378 & $1.424-20.306$ & 0.013 & 1.428 & $0.196-10.390$ & 0.725 \\
\hline Peritumoral LVD (< 10 vs $\geq 10)$ & 1.721 & $0.541-5.470$ & 0.358 & 0.572 & $0.046-7.043$ & 0.572 \\
\hline Peritumoral LVA (<3326 vs 3326) & 1.950 & $0.591-6.433$ & 0.273 & 1.230 & $0.136-11.125$ & 0.854 \\
\hline
\end{tabular}

HR Hazard ratio, CI Confidence interval, MVD Microvascular density, MVA Microvascular area, CSS Cancer-specific survival, LVA Lymph vessel area, LVD Lymph vessel density, AJCC American Joint Committee On Cancer, WHO/ISUP World Health Organization/International Society of Urological Pathology 
patients were significantly longer in patients with low intratumoral MVD or MVA than in patients with high intratumoral MVD or MVA, which corresponded with the result of a previous study [11]. Nevertheless, another recent study has revealed that high MVD was associated with longer survival, which contradicts the findings of our study [13]. Conclusively, MVD or MVA in the intratumoral area plays a minimal role in predicting the prognosis for Xp11.2 translocation RCC, but it is not an independent prognostic factor for Xp11.2 translocation RCC. Compared with the tumor microvessels in intratumoral and peritumoral areas of Xp11.2 translocation RCC, the tumor lymphangiogenesis was significantly different between intratumoral area and peritumoral area of Xp11.2 translocation RCC. Besides, findings from this work indicated a predominance of D2-40 positive lymph vessels primarily in the peritumoral area of Xp11.2 translocation RCC, whereas only 9 (26.5\%) cases depicted D2-40 positive lymph vessels in the intratumoral area of Xp11.2 translocation RCC. Recently, many studies have recorded intratumoral and peritumoral distribution of lymphatic vessels in RCC [10, 28]. Interestingly, the distribution of lymphatic vessels in Xp11.2 translocation RCC was consistent with that of traditional RCC. High-tissue pressure in tumor interstitium could be the main reason why lymph vessels were not detected in the intratumoral area of RCC [17]. Lastly, this study outlined that peritumoral LVD or LVA was not associated with CSS in patients with both low peritumoral LVD or LVA and high peritumoral LVD or LVA. However, previous reports indicated that RCC patients with low peritumoral LVD exhibited significantly shorter CSS [30]. Surprisingly, the role of tumor lymphangiogenesis in RCC remains elusive, and hence further studies are indispensable.

There are some limitations in the study. The sample size was not enough large due to the low incidence of this rare disease, and the follow-up time for patients was relatively short. In addition, the criteria for defining microvessels need to be further clarified, whereas the counting method should be more objective, accurate, convenient and efficient.

\section{Conclusions}

Overall, findings from this study indicate that Xp11.2 translocation RCC is a highly vascularized solid RCC, characterized by rich lymph vessels in the peritumoral area. Inhibiting tumor lymphangiogenesis and microangiogenesis would delay tumor growth and metastasis, which may provide novel strategies for treating Xp11.2 translocation RCC. Although MVD, MVA, LVD, or LVA cannot be used as novel risk factors predicting prognosis for Xp11.2 translocation RCC patients, high intratumoral MVA and MVD indicated poor prognosis and thereby they may be important to clinicians.

\section{Abbreviations}

RCC: Renal cell carcinoma; Xp11.2 translocation RCC: Xp11.2 translocation renal cell carcinoma; ccRCC: Clear cell renal cell carcinoma; MVD: Microvessel density; MVA: Microvessel area; LVD: Lymph vessel density; LVA: Lymph vessel density; CSS: Cancer-specific survival; PSM: Propensity score matching

\section{Acknowledgements}

Not applicable.

\section{Authors' contributions}

WLM and JY conducted the present study. WLM drafted the manuscript. WLM, JY, XZZ, NL, XHP, FQ, GTZ, XGL, HQG, LFX, WDG and DML collected clinical data. WLM and JY performed the statistical analyses. XZZ, NL, XHP, $F Q, G T Z, X G L, H Q G, L F X, W D G$ and DML participated in the study design and concept. NL and WDG revised the manuscript critically for intellectual content. All authors read and approved the final manuscript.

\section{Funding}

This research was supported by the National Natural Science Foundation of China (ID: 81572512), Nanjing SCI-TECH Development project (ID: 201803025) and Beijing Ronghe Medical Development Foundation. None of the funding bodies had any part in the design of the study and collection, analysis and interpretation of data, or in writing the manuscript.

\section{Availability of data and materials}

The datasets analyzed during the current study are available from the corresponding author on reasonable request.

\section{Ethics approval and consent to participate}

This study was reviewed and approved by the institutional ethics committees of Nanjing Drum Tower Hospital. The requirement for informed consent was waived given the retrospective nature of the data, that was applied by the ethics committee of Nanjing Drum Tower Hospital.

\section{Consent for publication}

Not applicable.

\section{Competing interests}

The authors declare that they have no competing interests.

\section{Author details}

'Department of Urology, Nanjing Drum Tower Hospital, The Affiliated Hospital of Nanjing University Medical School, No. 321 Zhongshan Road, Nanjing, Jiangsu Province, People's Republic of China 210008. ²Department of Pathology, Nanjing Drum Tower Hospital, The Affiliated Hospital of Nanjing University Medical School, Nanjing, Jiangsu, China. ${ }^{3}$ Immunology and Reproduction Biology Laboratory \& State Key Laboratory of Analytical Chemistry for Life Science, Medical School, Nanjing University, Nanjing, Jiangsu, China. ${ }^{4}$ Jiangsu Key Laboratory of Molecular Medicine, Nanjing University, Nanjing, Jiangsu, China.

Received: 29 August 2020 Accepted: 26 November 2020

Published online: 02 December 2020

References

1. Folkman J. What is the evidence that tumors are angiogenesis dependent? J Natl Cancer Inst. 1990;82:4-6.

2. Stacker SA, Achen MG, Jussila L, Baldwin ME, Alitalo K. Lymphangiogenesis and cancer metastasis. Nat Rev Cancer. 2002;2:573-83.

3. Verheul HM, Voest EE, Schlingemann RO. Are tumours angiogenesisdependent? J Pathol. 2004;202:5-13.

4. Folkman J, Watson K, Ingber D, Hanahan D. Induction of angiogenesis during the transition from hyperplasia to neoplasia. Nature. 1989;339:58-61.

5. Jussila L, Alitalo K. Vascular growth factors and lymphangiogenesis. Physiol Rev. 2002;82:673-700.

6. Wang XT, Xia QY, Zhou XJ, Rao Q. Xp11 translocation renal cell carcinoma and the Mesenchymal counterparts: an evolving concept with novel insights on Clinicopathologic features, prognosis, treatment, and classification. Crit Rev Oncog. 2017;22:481-97.

7. Mir MC, Trilla E, de Torres IM, Panizo A, Zlotta AR, Van Rhijn B, Morote J. Altered transcription factor E3 expression in unclassified adult renal cell 
carcinoma indicates adverse pathological features and poor outcome. BJU Int. 2011;108:E71-6.

8. Geller Jl, Argani P, Adeniran A, Hampton E, De Marzo A, Hicks J, Collins MH. Translocation renal cell carcinoma: lack of negative impact due to lymph node spread. Cancer. 2008;112:1607-16.

9. He J, Gan W, Liu S, Zhou K, Zhang G, Guo H, Zhu B. Dynamic computed tomographic features of adult renal cell carcinoma associated with Xp11.2 translocation/TFE3 gene fusions: comparison with clear cell renal cell carcinoma. J Comput Assist Tomogr. 2015;39:730-6.

10. Iwata T, Miyata Y, Kanda S, Nishikido M, Hayashi T, Sakai H, Kanetake H. Lymphangiogenesis and angiogenesis in conventional renal cell carcinoma: association with vascular endothelial growth factors a to D immunohistochemistry. Urology. 2008;71:749-54.

11. Sato M, Nakai Y, Nakata W, Yoshida T, Hatano K, Kawashima A, Fujita K, Uemura M, Takayama H, Nonomura N. Microvessel area of immature vessels is a prognostic factor in renal cell carcinoma. Int J Urol. 2014;21:130-4.

12. Sharma SG, Aggarwal N, Gupta SD, Singh MK, Gupta R, Dinda AK. Angiogenesis in renal cell carcinoma: correlation of microvessel density and microvessel area with other prognostic factors. Int Urol Nephrol. 2011:43:125-9.

13. Yildiz E, Ayan S, Goze F, Gokce G, Gultekin EY. Relation of microvessel density with microvascular invasion, metastasis and prognosis in renal cell carcinoma. BJU Int. 2008;101:758-64.

14. Chen X, Gan W, Ye Q, Yang J, Guo H, Li D. Application of polyclonal breakapart probes in the diagnosis of Xp11.2 translocation renal cell carcinoma. Zhonghua Yi Xue Za Zhi. 2014;94:3675-7.

15. Ma W, Liu N, Zhuang W, Li W, Qu F, Sun J, Xu W, Zhang L, Jia R, Xu L, et al. Comparative Clinicopathologic characteristics and outcomes of Paediatric and adult Xp11 translocation renal cell carcinomas: a retrospective multicentre study in China. Sci Rep. 2020;10:2249.

16. Choi Y, Park KJ, Ryu S, Kim DH, Yun J, Kang DK, Chun M. Papillary thyroid carcinoma involving cervical neck lymph nodes: correlations with lymphangiogenesis and ultrasound features. Endocr J. 2012;59:941-8.

17. Trojan L, Michel MS, Rensch F, Jackson DG, Alken P, Grobholz R. Lymph and blood vessel architecture in benign and malignant prostatic tissue: lack of lymphangiogenesis in prostate carcinoma assessed with novel lymphatic marker lymphatic vessel endothelial hyaluronan receptor (LYVE-1). J Urol. 2004;172:103-7.

18. Weidner N. Current pathologic methods for measuring intratumoral microvessel density within breast carcinoma and other solid tumors. Breast Cancer Res Treat. 1995:36:169-80.

19. Cheng SH, Liu JM, Liu QY, Luo DY, Liao BH, Li H, Wang KJ. Prognostic role of microvessel density in patients with renal cell carcinoma: a meta-analysis. Int J Clin Exp Pathol. 2014;7:5855-63.

20. MacLennan GT, Bostwick DG. Microvessel density in renal cell carcinoma: lack of prognostic significance. Urology. 1995;46:27-30.

21. Hemmerlein B, Kugler A, Ozisik R, Ringert RH, Radzun HJ, Thelen P. Vascular endothelial growth factor expression, angiogenesis, and necrosis in renal cell carcinomas. Virchows Arch. 2001;439:645-52.

22. Herbst C, Kosmehl H, Stiller KJ, Berndt A, Eiselt M, Schubert J, Katenkamp D. Evaluation of microvessel density by computerised image analysis in human renal cell carcinoma. Correlation to pT category, nuclear grade, proliferative activity and occurrence of metastasis. J Cancer Res Clin Oncol. 1998;124: $141-7$.

23. Lee JS, Jung JJ, Kim J. Quantification of angiogenesis by a computerized image analysis system in renal cell carcinoma. Anal Quant Cytol Histol. 2000; 22:469-74.

24. Imao T, Egawa M, Takashima $\mathrm{H}$, Koshida K, Namiki M. Inverse correlation of microvessel density with metastasis and prognosis in renal cell carcinoma. Int J Urol. 2004;11:948-53.

25. Sabo E, Boltenko A, Sova Y, Stein A, Kleinhaus S, Resnick MB. Microscopic analysis and significance of vascular architectural complexity in renal cell carcinoma. Clin Cancer Res. 2001;7:533-7.

26. Miyata Y, Kanda S, Ohba K, Nomata K, Eguchi J, Hayashida Y, Kanetake H. Tumor lymphangiogenesis in transitional cell carcinoma of the upper urinary tract: association with clinicopathological features and prognosis. J Urol. 2006;176:348-53.

27. Nativ O, Sabo E, Reiss A, Wald M, Madjar S, Moskovitz B. Clinical significance of tumor angiogenesis in patients with localized renal cell carcinoma. Urology. 1998;51:693-6.
28. Ozardili I, Guldur ME, Ciftci H, Bitiren M, Altunkol A. Correlation between lymphangiogenesis and clinicopathological parameters in renal cell carcinoma. Singap Med J. 2012;53:332-5.

29. Cao Y, Zhang ZL, Zhou M, Elson P, Rini B, Aydin H, Feenstra K, Tan MH, Berghuis $B$, Tabbey $R$, et al. Pericyte coverage of differentiated vessels inside tumor vasculature is an independent unfavorable prognostic factor for patients with clear cell renal cell carcinoma. Cancer. 2013;119:313-24.

30. Debinski P, Dembowski J, Kowal P, Szydelko T, Kolodziej A, Malkiewicz B, Tupikowski K, Zdrojowy R. The clinical significance of lymphangiogenesis in renal cell carcinoma. Med Sci Monit. 2013;19:606-11.

\section{Publisher's Note}

Springer Nature remains neutral with regard to jurisdictional claims in published maps and institutional affiliations.
Ready to submit your research? Choose BMC and benefit from:

- fast, convenient online submission

- thorough peer review by experienced researchers in your field

- rapid publication on acceptance

- support for research data, including large and complex data types

- gold Open Access which fosters wider collaboration and increased citations

- maximum visibility for your research: over $100 \mathrm{M}$ website views per year

At BMC, research is always in progress.

Learn more biomedcentral.com/submissions 